\title{
Dossier thématique : « Les Mots de l'histoire » - un bilan. Introduction
}

\section{Falk Bretschneider et Gudrun Gersmann}

\section{(2) OpenEdition}

\section{Journals}

Édition électronique

URL : http://journals.openedition.org/ifha/449

DOI : $10.4000 /$ ifha. 449

ISSN : 2198-8943

\section{Éditeur}

IFRA - Institut franco-allemand (sciences historiques et sociales)

\section{Édition imprimée}

Date de publication : 30 septembre 2012

Pagination : 152-155

ISSN : 2190-0078

\section{Référence électronique}

Falk Bretschneider et Gudrun Gersmann, «Dossier thématique : "Les Mots de l'histoire » - un bilan Introduction », Revue de l'IFHA [En ligne], 4 | 2012, mis en ligne le 14 février 2013, consulté le 30 avril 2019. URL : http://journals.openedition.org/ifha/449 ; DOI : 10.4000/ifha.449

Ce document a été généré automatiquement le 30 avril 2019.

(CIFHA 


\title{
Dossier thématique: " Les Mots de l'histoire » - un bilan. Introduction
}

\author{
Falk Bretschneider et Gudrun Gersmann
}

Ce dossier thématique reprend les contributions d'une journée d'études organisée le 15 juin 2012 à Paris, dans les locaux de l'Institut historique allemand (IHA), pour clore les huit ans du séminaire «Les Mots de l'histoire: historiens allemands et français face à leurs concepts et à leurs outils ", une initiative développée, dès 2004, par le Centre de recherches interdisciplinaires sur l'Allemagne (CRIA, UMR 8131, EHESS/CNRS) avec de nombreux partenaires. ${ }^{1}$ Visant à fédérer la recherche franco-allemande à Paris autour d'une réflexion d'ordre essentiellement historiographique et méthodologique, ce séminaire a rassemblé durant son existence un grand nombre de chercheurs, de doctorants et d'étudiants travaillant sur l'espace germanique ou utilisant une bibliographie en allemand en sciences humaines et sociales, et spécialement en histoire. Il s'est en outre appuyé sur un maillage dense de coopérations entre différentes institutions d'enseignements et de recherche, tant en ce qui concerne l'équipe d'enseignantschercheurs l'ayant animé que les doctorants et étudiants qui y ont participé.

Suivant une proposition faite par Marc Bloch en 1928, le séminaire a essayé de confronter de façon réflexive des concepts clés des sciences sociales et humaines françaises et allemandes depuis la fin du XIXe siècle et de faire porter l'attention notamment sur leurs transferts, (ré)appropriations, importations et exportations. Placé sous les paradigmes de l'histoire croisée et de l'interculturalité scientifique et historiographique, il a ainsi voulu concentrer le regard sur les temporalités, les délimitations disciplinaires et l'émergence d'objets de pensée cruciaux dans le champ des sciences humaines et sociales de part et d'autre du Rhin.

Les séances du séminaire, organisées à Paris une fois par mois pendant l'année académique, ont toutes suivi le même principe : faire dialoguer un intervenant français et un invité allemand autour d'une notion, d'un concept, d'un courant historiographique qu'il s'agissait d'examiner dans une approche croisée à partir des traditions et des définitions nationales et/ou disciplinaires propres. La présentation par chaque orateur 
invité était suivie d'un bref commentaire chargé de rassembler et de comparer les tendances et les perspectives présentées, préalable à une discussion générale au sein du séminaire. D'autres manifestations ont accompagné ces séances habituelles, notamment une série de journées d'études soit thématiques (p. ex. la journée d'études « Autour de la Lebenswelt ", 13 juin 2008) soit organisées pour permettre à des jeunes chercheurs de présenter leurs travaux en se fondant sur le modèle des « Mots de l'histoire » (la journée d'études « Junior », 10 juin 2011).

L'organisation du séminaire et des journées d'études s'est faite en étroite collaboration avec d'autres institutions éminentes de la coopération scientifique franco-allemande, à Paris et ailleurs: d'abord soutenu par le Centre interdisciplinaire d'études et de recherches sur l'Allemagne (CIERA) dans le cadre de ses "Programmes de formationrecherche ", le séminaire a obtenu, depuis 2008, un soutien financier régulièrement renouvelé de la part de l'Université franco-allemande (UFA). Il a été accueilli d'abord par la Maison de la recherche de Paris IV, le Centre d'histoire de Sciences Po et l'EHESS, avant de trouver, à partir de 2008, un lieu stable dans les locaux de l'Institut historique allemand de Paris, devenu co-organisateur du séminaire. Organisé pour promouvoir une fécondation réciproque des historiographies en France et en Allemagne, le séminaire a également obtenu le soutien du Centre Marc Bloch de Berlin et, surtout, de la Mission historique française en Allemagne à Göttingen devenue Institut français d'histoire en Allemagne à Francfort et qui a pris en charge, chaque année, une séance. La parution du présent dossier thématique dans la Revue de l'Institut est donc à la fois la suite logique d'une coopération de longue date et l'expression des liens étroits entre les différentes institutions porteuses du séminaire et l'IFHA et fait l'objet d'une brève présentation dans le numéro de la Revue.

Au-delà de ces coopérations institutionnelles, le séminaire avait également pour objectif de proposer une offre d'enseignement ouverte à l'ensemble des initiatives existantes de formation franco-allemande à la recherche en histoire (niveau Master et doctorat) dans l'environnement parisien avec des partenaires allemands. Cette fonction de rassembleur s'est d'abord exprimée à travers l'équipe d'enseignants-chercheurs qui animait le séminaire. Au noyau dur des organisateurs - dans un premier temps formé par Alexandre Escudier, Patrice Veit et Michael Werner et auxquels sont venus s'ajouter, au fil du temps, Falk Bretschneider, Christophe Duhamelle, Bernd Klesmann, Christine Lebeau et Pierre Monnet - se sont associés d'autres collègues, en tant que co-organisateurs ou membres de l'équipe pédagogique du séminaire: Claudine Delphis, Guillaume Garner, Jean-Louis Georget, Gudrun Gersmann, Franziska Heimburger, Wolfgang Kaiser, Régine Le Jan, Thomas Lienhard, Hélène Miard-Delacroix, Marie-Thérèse Mourey, Marie-Louise PelusKaplan. En outre, le séminaire s'est inséré dans les programmes pédagogiques proposés par les deux Collèges doctoraux franco-allemands EHESS/Université Humboldt de Berlin et Université Paris I/Université Goethe de Francfort, ainsi que les deux cursus intégrés franco-allemands EHESS/Université de Heidelberg et Université Paris VII/Université de Bielefeld.

Parmi toutes ces coopérations du séminaire, celle établie avec l'Institut historique allemand a été certainement la plus intense. Elle s'est inscrite d'emblée dans un ensemble d'autres initiatives communes, souvent encore en cours et développées en lien direct ou non avec le séminaire "Les Mots de l'histoire». Notons, à titre d'exemples, les onze volumes de l'Histoire franco-allemande, éditée depuis 2011 par Gudrun Gersmann et Michael Werner, ou une formation en paléographie allemande proposée depuis 2008 
conjointement par le CRIA et l'IHA. En outre, les deux institutions ont réuni leurs forces afin de proposer à la jeune recherche franco-allemande deux guides électroniques dont le premier volet, intitulé Faire de l'histoire en Allemagne: un guide pour les jeunes chercheurs français (sous la direction de Falk Bretschneider et Mareike König), est paru en 2011². Un deuxième volet, destiné à des jeunes chercheurs allemands désireux d'effectuer un séjour de recherche en France est prévu pour 2013, en coopération avec l'IFHA.

Dans leurs contributions, les auteurs du dossier reprennent et approfondissent les quelques aspects brièvement évoqués, dans cette introduction, de l'entreprise intellectuelle, institutionnelle et pédagogique que furent «Les Mots de l'histoire». Patrice Veit remonte d'abord aux origines pour retracer la dynamique du séminaire sous l'impulsion de ses évolutions théoriques, thématiques et institutionnelles. Christophe Duhamelle présente ensuite une analyse des choix thématiques respectifs opérés par les organisateurs, en insistant sur le fait que dans les manières de dire s'expriment toujours des manières de faire, permettant à travers un retour réflexif sur soi d'aller au-delà des apparentes évidences qui structurent un champ de recherche. Reprenant l'idée des "Mots de l'histoire » comme laboratoire de réflexivité, la contribution suivante, celle de Michael Werner, revient sur les rapports du séminaire avec les approches de la sémantique historique ou Begriffsgeschichte, en mettant en évidence à la fois les effets bénéfiques et les limites d'une approche destinée à historiciser le travail des historiens en mettant en perspective historique leurs outils analytiques et discursifs. Pierre Monnet, quant à lui, met en exergue le rôle fondamental que le séminaire a pu jouer pour la constitution d'un maillage dense de coopérations scientifiques franco-allemandes au moment où se déroulaient d'importants changements par l'accélération des réformes et des remembrements du paysage scientifique et académique des deux côtés du Rhin. Anna Karla, enfin, évoque un autre élément central des "Mots de l'histoire ", à savoir le souci permanent d'intégrer dans les travaux du séminaire doctorants et étudiants issus des formations franco-allemandes présentes à Paris, et sur le décalage existant entre la perception qu'ils se font eux-mêmes de leur position dans le système de la recherche et les conceptions officielles que la politique leur oppose en les désignant comme «jeunes chercheurs » ou « Nachwuchswissenschaftler ».

\section{NOTES}

1. Pour un résumé de la journée d'études cf. l'article d'Axel Dröber : "Ehre und Honneur sind nicht das gleiche. Deutsch-französische Begriffsgeschichte bei den 'Mots' ", in: Frankfurter Allgemeine Zeitung (25.7.2012).

2. Cf. Falk Bretschneider et Mareike König : «Faire de l'histoire en Allemagne. Un guide pour les jeunes chercheurs français ", in : Revue de l'Institut français d'histoire en Allemagne 3 (2011), p. 89-94. 


\section{AUTEURS}

\section{FALK BRETSCHNEIDER}

Falk Bretschneider est maître de conférences à l'École des hautes études en sciences sociales (Paris).

\section{GUDRUN GERSMANN}

Gudrun Gersmann est professeure d'histoire moderne à l'université de Cologne et fut, de 2007 à 2012, directrice de l'Institut historique allemand à Paris. 